\title{
Diagonal Riccati Stability and Positive Time-Delay Systems
}

\author{
Oliver Mason ${ }^{\mathrm{a}, 1, *}$ \\ ${ }^{a}$ Hamilton Institute, National University of Ireland, Maynooth \\ Maynooth, Co. Kildare, Ireland
}

\begin{abstract}
We consider a class of algebraic Riccati equations arising in the study of positive linear time-delay systems. We show that this class admits diagonal positive definite solutions. This implies that exponentially stable positive linear timedelay systems possess Lyapunpov-Krasovskii functionals of a simple quadratic form. We also show that for this class of equations, the existence of positivedefinite solutions is equivalent to a simple spectral condition on the coefficient matrices.
\end{abstract}

Keywords: Riccati equations; positive time-delay systems; diagonal Lyapunov functions.

This appeared in Systems Control Letters 61 (2012), pp. 6-10;

DOI:10.1016/j.sysconle.2011.09.022

\section{Introduction}

The algebraic Riccati equation (ARE) arises in various guises in numerous areas of Control Theory [20, 14]. In particular, the role of the symmetric and hermitian ARE in the theory of $H^{\infty}$ control and its connection with the bounded real lemma are well known [5]. The ARE also arises in characterising complex stability radii for linear time-invariant (LTI) systems [15], and in the construction of Lyapunov-Krasovskii functionals for linear time-delay systems [18]. The work of this short note is motivated by the connection between the ARE and the stability of linear time-delay systems.

In particular, Theorem 3.1, the main result of this paper, addresses the following problem, which was posed in [18]. Characterise those pairs of matrices $A, B$ in $\mathbb{R}^{n \times n}$ for which the continuous algebraic Riccati inequality (ARI)

$$
A^{T} P+P A+Q+P B Q^{-1} B^{T} P \prec 0
$$

\footnotetext{
*Corresponding author. Tel.: +353 (0)1 7086274; fax: +353 (0)1 7086269; email: oliver.mason@nuim.ie

${ }^{1}$ Supported by the Irish Higher Educational Authority(HEA) PRTLI Network Mathematics Grant and Science Foundation Ireland Grant 08-RFP-ENE1417
} 
admits positive definite solutions $P, Q$. Throughout the paper, $X \prec 0$ denotes that $X=X^{T}$ is negative definite.

A major motivation for considering this problem arises in the study of the linear time-delay system

$$
\dot{x}=A x(t)+B x(t-\tau) .
$$

In fact, if $P, Q$ satisfy (1), then the functional

$$
V(\phi)=\phi(0)^{T} P \phi(0)+\int_{-\tau}^{0} \phi(s)^{T} Q \phi(s) d s
$$

defined on the space of continuous functions on $[-\tau, 0]$ defines a LyapunovKrasovskii functional [19] for the system (2) for every $\tau \geq 0$. The existence of such a functional implies that the zero equilibrium of (2) is exponentially stable (in an abuse of notation, we say that the system (2) is exponentially stable when its zero equilibrium is exponentially stable). Note that it is not in general true that any exponentially stable system (2) possesses a Lyapunov-Krasovskii functional of this simple form.

Positive systems, for which non-negative initial conditions give rise to nonnegative evolutions arise in applications ranging from Biology to Economics $[2,3,17,7]$. It is known [7] that the time-delay system (2) is positive if and only if

(i) $A$ is Metzler meaning that $a_{i j} \geq 0$ for $i \neq j$;

(ii) $B$ is a non-negative matrix meaning $b_{i j} \geq 0$ for all $i, j$.

The stability properties of positive linear delay systems have been extensively studied recently (see for example $[7,8,9,10,11,12,17]$ ). In particular, the results of $[17,7]$ show that the following statements are equivalent:

(a) (2) is exponentially stable for $\tau=0$;

(b) there exists an entry-wise positive vector $v$ such that $V(\phi)=v^{T} \phi(0)+$ $\int_{-\tau}^{0} v^{T} B \phi(s) d s$ is a Lyapunov-Krasovskii functional for (2) for every $\tau \geq 0$;

(c) (2) is exponentially stable for every $\tau \geq 0$.

Under the assumption that the delayed system (2) is positive, in Theorem 3.1 we provide simple conditions for (1) to admit positive definite solutions $P, Q$. In fact, we show that such solutions exist if and only if the matrix $A+B$ is Hurwitz (meaning all of its eigenvalues lie in the open left half plane). Moreover, we show that the matrix $P$ can be chosen to be diagonal in this case. In view of the remarks made above, this result implies that conditions (a), (b), (c) above are also equivalent to the existence of a diagonal Lyapunov-Krasovskii functional of the form (3) for (2). Thus, Theorem 3.1 shows that a key property of exponentially stable positive linear time-invariant (LTI) systems, namely the existence of diagonal Lyapunov functions, extends to positive linear time-delay systems. 
The layout of the paper is as follows. In Section 2, we establish common notation and recall some necessary results from convex analysis and matrix theory. In Section 3 the main result of the paper concerning the existence of diagonal solutions to (1) is presented and proven. In Section 4, we discuss some implications of Theorem 3.1 and describe a counterexample to one potential generalisation of this result. Finally, in Section 5, we present our conclusions.

\section{Background}

Throughout the paper, $\mathbb{R}$ and $\mathbb{R}^{n}$ denote the field of real numbers and the vector space of all $n$-tuples of real numbers, respectively. $\mathbb{R}^{n \times n}$ denotes the space of $n \times n$ matrices with real entries. For $x \in \mathbb{R}^{n}$ and $i=1, \ldots, n, x_{i}$ denotes the

$i^{\text {th }}$ coordinate of $x$. Similarly, for $A \in \mathbb{R}^{n \times n}, a_{i j}$ denotes the $(i, j)^{t h}$ entry of $A$. For a vector $x \in \mathbb{R}^{n}: x \geq 0$ means that $x_{i} \geq 0$ for $1 \leq i \leq n ; x>0$ means that $x \geq 0$ and $x_{i}>0$ for some $i ; x \gg 0$ means that $x_{i}>0$ for $1 \leq i \leq n$. The notations $x \leq 0, x<0$ and $x \ll 0$ are defined in the obvious way.

We denote the $n \times n$ matrix all of whose entries are equal to one by $\mathbf{1}_{n \times n}$.

The space of symmetric matrices in $\mathbb{R}^{n \times n}$ is denoted by $\operatorname{Sym}(n, \mathbb{R})$. We use the notation $A \succ 0(A \succeq 0)$ to denote that the matrix $A \in \operatorname{Sym}(n, \mathbb{R})$ is positive definite (semi-definite). The notation $A \prec 0,(A \preceq 0)$ denotes that $A$ is negative definite (semi-definite).

For $A \in \mathbb{R}^{n \times n}$, we denote the spectrum of $A$ by $\sigma(A)$. The spectral radius of $A$ is denoted by $\rho(A)$. Also, the notation $\mu(A)$ denotes the spectral abscissa of $A$ which is defined as follows:

$$
\mu(A):=\max \{\operatorname{Re}(\lambda): \lambda \in \sigma(A)\} .
$$

A matrix $A$ for which $\mu(A)<0$ is said to be Hurwitz, while $A$ is said to be Schur-Cohn if $\rho(A)<1$.

A nonnegative matrix $A$ is irreducible if for every nonempty proper subset $K$ of $N:=\{1, \cdots, n\}$, there exists an $i \in K, j \in N \backslash K$ such that $a_{i j}>0$. The next result concerning Metzler matrices is standard and follows from the Perron-Frobenius theorem [6].

Theorem 2.1. Let $A \in \mathbb{R}^{n \times n}$ be Metzler. Then $\mu(A) \in \sigma(A)$. In addition, there exist vectors $v \geq 0, w \geq 0$ such that $v^{T} A=\mu(A) v^{T}, A w=\mu(A) w$.

Let $E$ be a finite-dimensional Euclidean space equipped with an inner product $\langle\cdot, \cdot\rangle$. The following well-known result on separation of convex sets in $E$ shall be needed in the next section. Recall that a subset $C$ of $E$ is a cone if for all $t>0, x \in C$, we have $t x \in C$.

Proposition 2.1. [16] Let $C_{1}, C_{2}$ be non-empty convex subsets of E. Further, assume that $C_{2}$ is a cone and $C_{1} \cap C_{2}$ is empty. Then there exists a non-zero vector $v \in E$ such that

$$
\begin{aligned}
& \langle v, x\rangle \geq 0 \text { for all } x \in C_{1} \\
& \langle v, x\rangle \leq 0 \text { for all } x \in C_{2} .
\end{aligned}
$$


In the next Section, we shall apply the above result to convex sets in the Euclidean space $\operatorname{Sym}(n, \mathbb{R})$ of $n \times n$ real symmetric matrices equipped with the inner product

$$
\langle A, B\rangle=\operatorname{trace}(A B)
$$

\section{Diagonal Riccati stability and positive linear time-delay systems}

The main result of this section establishes that if $A \in \mathbb{R}^{n \times n}$ is Metzler and $B$ is nonnegative, then there exists a positive definite diagonal matrix $D$ and a positive definite matrix $Q$ satisfying the Riccati inequality

$$
A^{T} D+D A+Q+D B Q^{-1} B^{T} D \prec 0
$$

if and only if $A+B$ is Hurwitz.

We next recall three technical lemmas, which shall prove useful in proving this result. The first of these concerns the existence of common diagonal Lyapunov functions for positive LTI systems and a proof can be found in [22].

Lemma 3.1. Let $A \in \mathbb{R}^{n \times n}$ be Metzler and $B \in \mathbb{R}^{n \times n}$ be nonnegative. If $A+B$ is Hurwitz, then there exists a positive definite diagonal matrix $D$ satisfying

$$
\begin{aligned}
& A^{T} D+D A \prec 0 \\
& (A+B)^{T} D+D(A+B) \prec 0
\end{aligned}
$$

Recall that for any $H=H^{T} \succeq 0$ in $\mathbb{R}^{n \times n}, h_{i j}^{2} \leq h_{i i} h_{j j}$. The following fact, which also follows from Lemma 2 in [21], is an easy consequence of this observation.

Lemma 3.2. Let $A \in \mathbb{R}^{n \times n}$ be Metzler and let $H=H^{T} \succeq 0$ be positive semidefinite. Define the vector $v \in \mathbb{R}^{n}$ by $v_{i}=\sqrt{h_{i i}}$ for $1 \leq i \leq n$. Then

$$
\operatorname{trace}\left[v v^{T} A\right] \geq \operatorname{trace}[H A] .
$$

The next result is based on the Schur complement and follows from Theorem 7.7.6 in [4].

Lemma 3.3. Let $A \in \mathbb{R}^{n \times n}, B \in \mathbb{R}^{n \times n}$ be given. Then the matrices $P \succ 0$, $Q \succ 0$ in $\mathbb{R}^{n \times n}$ satisfy (1) if and only if:

$$
S:=\left(\begin{array}{cc}
A^{T} P+P A+Q & P B \\
B^{T} P & -Q
\end{array}\right) \prec 0 .
$$

Theorem 3.1. Let $A \in \mathbb{R}^{n \times n}$ be Metzler and $B \in \mathbb{R}^{n \times n}$ be nonnegative. Then there exists a positive definite diagonal matrix $D$ and a positive definite matrix $Q$ satisfying the Riccati inequality (4) if and only if $A+B$ is Hurwitz. 
Proof: First assume that there exists a positive definite diagonal $D$ and a positive definite $Q$ satisfying (4). From Lemma 3.3, it follows that for any $x \neq 0$ in $\mathbb{R}^{n}$,

$$
\left(x^{T}, x^{T}\right)\left(\begin{array}{cc}
A^{T} D+D A+Q & D B \\
B^{T} D & -Q
\end{array}\right)\left(\begin{array}{l}
x \\
x
\end{array}\right)<0
$$

which implies that $x^{T}\left(A^{T} D+D A+B^{T} D+D B\right) x<0$. Hence

$$
(A+B)^{T} D+D(A+B) \prec 0
$$

and it follows from the classical Lyapunov theorem [6] that $A+B$ is Hurwitz. Conversely assume that $A+B$ is Hurwitz. As $A$ is Metzler and $B$ is nonnegative, it follows from Lemma 3.1 that there exists a positive definite diagonal matrix $D$ satisfying (5). Choose some such $D$. To complete the proof, we shall show that, for this $D$, there exists a $Q \succ 0$ such that

$$
\left(\begin{array}{cc}
A^{T} D+D A+Q & D B \\
B^{T} D & -Q
\end{array}\right) \prec 0 .
$$

By way of contradiction, suppose that no such $Q$ exists. Consider the sets

$$
\begin{gathered}
C_{1}:=\left\{\left(\begin{array}{cc}
A^{T} D+D A+Q & D B \\
B^{T} D & -Q
\end{array}\right): Q \succ 0, Q \in \operatorname{Sym}(n, \mathbb{R})\right\}, \\
C_{2}:=\{X \in \operatorname{Sym}(2 n, \mathbb{R}): X \prec 0\} .
\end{gathered}
$$

By assumption, $C_{1} \cap C_{2}=\emptyset$. It is immediate that the set $C_{2}$ is a convex cone in the Euclidean space $\operatorname{Sym}(2 n, \mathbb{R})$, while a straightforward calculation shows that $C_{1} \subset \operatorname{Sym}(2 n, \mathbb{R})$ is non-empty and convex. Hence from Proposition 2.1, there exists some non-zero $H \in \operatorname{Sym}(2 n, \mathbb{R})$ such that

$$
\langle H, X\rangle \geq 0 \quad \forall X \in C_{1},
$$

while

$$
\langle H, X\rangle \leq 0 \quad \forall X \in C_{2} .
$$

By continuity, (9) implies that $\langle H, X\rangle \geq 0$ for all $X \succeq 0$ in $\operatorname{Sym}(2 n, \mathbb{R})$. Noting that for every $x \in \mathbb{R}^{2 n}, X=x x^{T} \succeq 0$, it follows that $H \succeq 0$. (See also Corollary 7.5.4 of [4].)

Now partition $H$ as

$$
H=\left(\begin{array}{ll}
H_{11} & H_{12} \\
H_{12}^{T} & H_{22}
\end{array}\right),
$$

where $H_{11}, H_{22}$ are in $\operatorname{Sym}(n, \mathbb{R})$ and $H_{12} \in \mathbb{R}^{n \times n}$. Equation (8) implies that

$$
\operatorname{trace}\left[\left(\begin{array}{cc}
H_{11} & H_{12} \\
H_{12}^{T} & H_{22}
\end{array}\right)\left(\begin{array}{cc}
A^{T} D+D A+Q & D B \\
B^{T} D & -Q
\end{array}\right)\right] \geq 0
$$


for all $Q \succ 0$. Expanding this equation,we see that

$$
\operatorname{trace}\left[H_{11}\left(A^{T} D+D A\right)+H_{12} B^{T} D+H_{12}^{T} D B\right]+\operatorname{trace}\left[H_{11} Q-H_{22} Q\right] \geq 0
$$

for all $Q \succ 0$. Rearranging the above inequality, it follows that for all $Q \succ 0$ we must have

$$
\operatorname{trace}\left[H_{11}\left(A^{T} D+D A\right)+H_{12} B^{T} D+H_{12}^{T} D B\right] \geq \operatorname{trace}\left[Q\left(H_{22}-H_{11}\right)\right] .
$$

Now if trace $\left[Q\left(H_{22}-H_{11}\right)\right]>0$ for any $Q \succ 0$, we can choose $t>0$ sufficiently large to ensure that trace $\left[t Q\left(H_{22}-H_{11}\right)\right]$ is greater than trace $\left[H_{11}\left(A^{T} D+\right.\right.$ $\left.D A)+H_{12} B^{T} D+H_{12}^{T} D B\right]$. It follows that the right hand side of (11) must be non-positive for all $Q \succ 0$. Furthermore, if

$$
\operatorname{trace}\left[H_{11}\left(A^{T} D+D A\right)+H_{12} B^{T} D+H_{12}^{T} D B\right]<0
$$

then we could choose $t>0$ sufficiently small to ensure

$$
\operatorname{trace}\left[t Q\left(H_{22}-H_{11}\right)\right]>\operatorname{trace}\left[H_{11}\left(A^{T} D+D A\right)+H_{12} B^{T} D+H_{12}^{T} D B\right] .
$$

Putting these observations together, we see that

$$
\begin{gathered}
\operatorname{trace}\left[Q\left(H_{22}-H_{11}\right)\right] \leq 0 \quad \text { for all } Q \succ 0 \\
\operatorname{trace}\left[H_{11}\left(A^{T} D+D A\right)+H_{12} B^{T} D+H_{12}^{T} D B\right] \geq 0
\end{gathered}
$$

For $1 \leq i \leq 2 n$, write $h_{i i}$ for the $i$ th diagonal element of the full matrix $H$ in (10). Define the vector $v \in \mathbb{R}^{2 n}$ by $v_{i}=\sqrt{h_{i i}}$ for $1 \leq i \leq 2 n$ and write $v^{T}=\left[u^{T}, w^{T}\right]$ for $u \in \mathbb{R}^{n}, w \in \mathbb{R}^{n}$. Then $u_{i}^{2}$ is the $i^{t h}$ entry along the main diagonal of the matrix $H_{11}$ and $w_{i}^{2}$ is the $i^{t h}$ entry along the main diagonal of the matrix $H_{22}$. It follows from (12) that $H_{22}-H_{11} \preceq 0$ and thus $u_{i} \geq w_{i} \geq 0$ for $1 \leq i \leq n$. Moreover, $u \neq 0$ as otherwise all diagonal entries of $H_{11}, H_{22}$ would be zero and hence, as $H_{11}, H_{22}$ and $H$ are positive semi-definite, it would follow that $H=0$, which is a contradiction.

Next note that

$\operatorname{trace}\left[H_{11}\left(A^{T} D+D A\right)+H_{12} B^{T} D+H_{12}^{T} D B\right]=\operatorname{trace}\left[H\left(\begin{array}{cc}A^{T} D+D A & D B \\ B^{T} D & 0\end{array}\right)\right]$.

As $H$ is positive semi-definite and the matrix

$$
\left(\begin{array}{cc}
A^{T} D+D A & D B \\
B^{T} D & 0
\end{array}\right)
$$

is Metzler, it follows from Lemma 3.2 that

$$
\operatorname{trace}\left[v v^{T}\left(\begin{array}{cc}
A^{T} D+D A & D B \\
B^{T} D & 0
\end{array}\right)\right] \geq \operatorname{trace}\left[H\left(\begin{array}{cc}
A^{T} D+D A & D B \\
B^{T} D & 0
\end{array}\right)\right] .
$$


Combining (14), (15) and (13), we see that

$$
\operatorname{trace}\left[v v^{T}\left(\begin{array}{cc}
A^{T} D+D A & D B \\
B^{T} D & 0
\end{array}\right)\right] \geq 0 .
$$

Now recall that $v^{T}=\left[u^{T}, w^{T}\right]$ with $u_{i} \geq w_{i} \geq 0$ for $1 \leq i \leq n$ and $u \neq 0$. Expanding equation (16), we see that

$$
\operatorname{trace}\left[u u^{T}\left(A^{T} D+D A\right)+u w^{T} B^{T} D+w u^{T} D B\right] \geq 0
$$

which is equivalent to

$$
u^{T} D A u+u^{T} D B w \geq 0 .
$$

But, we know that $B$ and $D$ are both nonnegative, and moreover $u_{i} \geq w_{i} \geq 0$ for $1 \leq i \leq n$. Thus, it follows from (18) that

$$
u^{T}(D A+D B) u \geq 0
$$

which implies that

$$
u^{T}\left(A^{T} D+D A+B^{T} D+D B\right) u \geq 0
$$

contradicting the fact that $D$ satisfies (5). This contradiction shows that there must exist a matrix $Q \succ 0$ satisfying (7) and hence that there exists a $Q \succ 0$ such that $D, Q$ satisfy the Riccati inequality (4). This completes the proof.

\section{Riccati stability, common Lyapunov solutions and the stability of positive linear time-delay systems}

We next describe the connection of Theorem 3.1 to known results relating the spectral properties of $A, B$ to the existence of solutions to (1).

In [18], the following necessary condition for the existence of positive definite solutions to (1) was given.

Theorem 4.1. If there exist positive definite matrices $P, Q$ satisfying (1), then $A$ is Hurwitz and $A^{-1} B$ is Schur-Cohn.

As noted in [18], no complete converse of this theorem is known. In Theorem 2 and Theorem 3 of [18], partial converses are described. First, it is shown that if $A^{-1} B$ is Schur-Cohn, then by pre-multiplying $A$ and $B$ by a suitable orthogonal matrix we obtain a pair of matrices admitting positive definite solutions to the associated Riccati inequality. Second, for any Hurwitz $A$, there exists a matrix $B$ such that $A^{-1} B$ is Schur-Cohn and (1) has positive definite solutions. We use Theorem 3.1 to show in Corollary 4.1 that if $A$ is Metzler and $B$ is non-negative, the converse of Theorem 4.1 is true. We first present a simple lemma concerning nonnegative matrices, whose proof is included in the interest of completeness.

Lemma 4.1. Let $T \in \mathbb{R}^{n \times n}$ be nonnegative. Suppose there exists some $w>0$ such that $T w \geq w$. Then $\rho(T) \geq 1$. 
Proof: By way of contradiction, suppose that $\rho(T)<1$. By continuity of eigenvalues, $\rho\left(T+\delta \mathbf{1}_{n \times n}\right)<1$ for sufficiently small $\delta>0$. Moreover, $T+\delta \mathbf{1}_{n \times n}$ is irreducible. From the classical Perron-Frobenius Theorem [4], it follows that there exists some vector $v \gg 0$ with

$$
v^{T}\left(T+\delta \mathbf{1}_{n \times n}\right)=\rho\left(T+\delta \mathbf{1}_{n \times n}\right) v^{T} \ll v^{T} .
$$

This implies that $v^{T}\left(T+\delta \mathbf{1}_{n \times n}\right) w<v^{T} w$. However as $v \gg 0$ and $T w \geq w$, we must have

$$
v^{T}\left(T+\delta \mathbf{1}_{n \times n}\right) w \geq v^{T} T w \geq v^{T} w .
$$

This contradiction shows that $\rho(T) \geq 1$ as claimed.

Corollary 4.1. Let $A \in \mathbb{R}^{n \times n}$ be Metzler and let $B \in \mathbb{R}^{n \times n}$ be nonnegative. Assume that $A$ is Hurwitz and $A^{-1} B$ is Schur-Cohn. Then there exist positive definite matrices $P, Q$ satisfying (1).

Proof: We shall show that the assumptions that $A$ is Hurwitz and $A^{-1} B$ is Schur-Cohn imply that $A+B$ is Hurwitz. The result then follows from Theorem 3.1.

By way of contradiction, assume that $A+B$ is not Hurwitz. It follows from Theorem 2.1 that there exists a non-zero vector $v \geq 0$ and some real number $\lambda \geq 0$ with

$$
\begin{aligned}
(A+B) v & =\lambda v \\
\Rightarrow\left(I+A^{-1} B\right) v & =\lambda A^{-1} v \\
\Rightarrow\left(-A^{-1} B\right) v & =v-\lambda A^{-1} v .
\end{aligned}
$$

As $A$ is Metzler and Hurwitz, $-A^{-1}$ is nonnegative ([6], Chapter 2); hence $-A^{-1} B$ is nonnegative and moreover $-\lambda A^{-1} v \geq 0$. Thus, it follows from (20) that

$$
\left(-A^{-1} B\right) v \geq v .
$$

It now follows from Lemma 4.1 that $-A^{-1} B$ and hence $A^{-1} B$ is not SchurCohn. This contradiction shows that $A+B$ must be Hurwitz. The result now follows from Theorem 3.1.

In the proof of Theorem 3.1, we have shown that for any positive definite diagonal $D$ satisfying the Lyapunov inequalities $A^{T} D+D A \prec 0,(A+B)^{T} D+D(A+$ $B) \prec 0$ there exists a positive definite $Q$ such that $D, Q$ satisfy (4). Moreover, the first part of the proof can be readily adapted to show that if there exist positive definite matrices $P, Q$ satisfying (1), then $P$ must satisfy

$$
\begin{aligned}
A^{T} P+P A & \prec 0 \\
(A+B)^{T} P+P(A+B) & \prec 0
\end{aligned}
$$

In view of these observations, it may be tempting to conjecture that the existence of $P \succ 0$ satisfying (22) is also sufficient for the existence of positive definite solutions to (1) for general $A, B$. However, this is not the case as is made clear by the following example. 
Example 4.1. Consider the matrices $A, B$ in $\mathbb{R}^{2 \times 2}$ given by

$$
A=\left(\begin{array}{ll}
-3 & 2 \\
-3 & 1
\end{array}\right), B=\left(\begin{array}{ll}
1 & -1 \\
1 & -2
\end{array}\right)
$$

It is simple to verify that neither of the matrices $A^{-1}(A+B), A(A+B)$ has any real, negative eigenvalues. It follows from the results of [13] that there exists a positive definite matrix $P$ satisfying (22). Now consider the matrix $H \in \operatorname{Sym}(4, \mathbb{R})$ in the block form (10) with

$$
H_{11}=\left(\begin{array}{cc}
7.9 & 10.6 \\
10.6 & 16.9
\end{array}\right), H_{22}=\left(\begin{array}{cc}
6.7 & 9.5 \\
9.5 & 14.9
\end{array}\right), H_{12}=\left(\begin{array}{cc}
-1.2 & -4.2 \\
-5.2 & -10.8
\end{array}\right)
$$

The following can be verified by direct computation.

(i) $H>0$;

(ii) $H_{11}-H_{22}>0$;

(iii) $A H_{11}+H_{11} A^{T}+B H_{12}^{T}+H_{12} B^{T}>0$.

It follows immediately from points (ii) and (iii) that for all positive definite $P, Q$ we must have

$$
\begin{array}{r}
\operatorname{trace}\left[P\left(A H_{11}+H_{11} A^{T}+H_{12} B^{T}+B H_{12}^{T}\right)+Q\left(H_{11}-H_{22}\right)\right]>0 \\
\left.\Rightarrow \operatorname{trace}\left[H_{11}\left(P A+A^{T} P\right)+H_{12} B^{T} P+H_{12}^{T} P B\right)+Q\left(H_{11}-H_{22}\right)\right]>0 \\
\Rightarrow \text { trace }\left[\left(\begin{array}{cc}
H_{11} & H_{12} \\
H_{12}^{T} & H_{22}
\end{array}\right)\left(\begin{array}{cc}
A^{T} P+P A+Q & P B \\
B^{T} P & -Q
\end{array}\right)\right]>0 .
\end{array}
$$

As $H>0$, this immediately implies that there exist no positive definite matrices $P, Q$ satisfying (6) and hence that no positive definite solutions exist to the Riccati inequality (4).

\section{Concluding remarks}

Motivated by a question posed in [18], we have considered a form of the algebraic Riccati inequality (ARI) that arises in the stability theory of linear time-delay systems. Specifically, for positive linear time-delay systems, we have given a simple necessary and sufficient condition for the corresponding ARI to possess positive definite solutions. Moreover, we have shown that in this case, the matrix $P$ in (1) can be chosen to be diagonal. Combining this result with previous work in $[7,17]$, we see that the stability properties of positive linear time-delay systems mirror those of positive LTI systems in a straightforward manner.

It is natural to ask if Theorem 3.1 can be extended beyond the class of Metzler $A$ and nonnegative $B$. Example 4.1 shows that the existence of a common Lyapunov solution for the matrices $A, A+B$ is not in general sufficient for (1) to have positive definite solutions. This of course implies that Theorem 3.1 is 
not true in general. However, it is worth investigating whether or not a similar result may hold under stronger conditions such as the existence of a common

diagonal Lyapunov solution. Hopefully, some results along these lines will be obtained in the near future.

\section{Acknowledgements}

The author would like to thank the editor and anonymous reviewers for their helpful suggestions, which have helped improve the quality of the manuscript.

\section{References}

[1] Farina, L. and Rinaldi, S. Positive Linear Systems. Theory and Applications. Pure and Applied mathematics. John Wiley \& Sons, Inc., Newyork, NY, USA, 2000.

[2] Fornasini, E. and Valcher, M. E.. Linear copositive Lyapunov functions for continuous-time positive switched systems. IEEE Transactions on Automatic Control, 55(8) (2010) 1933-1937

[3] Hernandez-Vargas, E., Colaneri, P., Middleton, R. and Blanchini, F. Discrete-time control for switched positive systems with application to mitigating viral escape. International Journal of Robust and Nonlinear Control, 21 (2011) 1093-1011

[4] Horn, R.A. and Johnson, C.R. Matrix Analysis. Cambridge University Press, New York, 1985.

[5] Lancaster, P. and Rodman, L. Algebraic Riccati Equations. Oxford University Press, New York, 1995.

[6] Horn, R.A. and Johnson, C.R. Topics in Matrix Analysis. Cambridge University Press, New York, 1991.

[7] Haddad W. M., Chellaboina V. Stability theory for nonnegative and compartmental dynamical systems with time delay. Systems and Control Letters, 51 (2004) 355-361.

[8] Ait Rami, M. Stability Analysis and Synthesis for Linear Positive Systems with Time-Varying Delays Proceedings of the 3rd International Symposium on Positive Systems, Lecture Notes in Control and Information Sciences, Springer-Verlag Volume 389 (2005), 205-215.

[9] Anh, B. T., Son, N. K. and Thanh, D. D. X. Stability radii of positive linear time-delay systems under fractional perturbations Systems Control Letters, 58 (2009) 155-159 
[10] Buslowicz, M. and Kaczorek, T. Robust stability of positive discrete-time interval systems with time delays Bull. Pol. Acad. Sci. Tech. Sci., 52(2) (2004) 99-102.

[11] De La Sen, M. On positivity and stability of a class of time-delay systems Nonlinear Analysis: Real World Applications, 8(3) (2007) 749-768

[12] Wu,L., Lam, J., Shu, Z. and Du, B. On stability and stabilizability of positive delay systems Asian Journal of Control, 11(2) (2009) 226-234

[13] Shorten, R. and Narendra, K. Necessary and Sufficient Conditions for the Existence of CQLFs for M Stable Second Order Linear Systems International Journal of Adaptive Control and Signal Processing, 16(10) (2002) 709-728

[14] Wimmer, H. The algebraic Riccati equation: conditions for the existence and uniqueness of solutions Linear algebra and its applications, 58 (1984) $441-452$.

[15] Hinrichsen D. and Pritchard, A.J. Stability radius for structured perturbations and the algebraic Riccati equation. Systems and Control Letters, 8 (1986) 105-113.

[16] Barvinok, A. A course in convexity American Mathematical Society, Rhode Island, 2002

[17] Ngoc, P.H.A. A Perron-Frobenius theorem for a class of positive quasipolynomial matrices Applied Mathematics Letters, 19 (2006) 747-751.

[18] Verriest, E.I. Riccati stability in: V.D. Blondel, A. Megretski (Eds.), Unsolved Problems in Mathematical Systems and Control Theory, Princeton University Press (2004) 49-53

[19] Hale, J.K and Verduyn Lunel, S.M. Introduction to Functional Differential Equations. Springer-Verlag, 1993.

[20] Abou-Kandil, H., Freiling, G., Ionescu, V.and Jank, G. Matrix Riccati Equations in Control and Systems Theory. Birkhauser, 2003.

[21] Ebihara, Y., Peaucelle, D., Arzelier, D., Hagiwara, T and Oishi, Y. Dual LMI approach to linear positive system analysis IEEE Conference on Decision and Control, Orlando, 2011

[22] Mason, O. and Shorten, R. On the simultaneous diagonal stability of a pair of positive linear systems Linear Algebra and its Applications, 413 (2006), 13-23 